\title{
High spatial and temporal resolution observations of an impulse-driven field line resonance in radar backscatter artificially generated with the Tromsø heater
}

\author{
T. K. Yeoman ${ }^{1}$, D. M. Wright ${ }^{1}$, T. R. Robinson ${ }^{1}$, J. A. Davies ${ }^{1}$, M. Rietveld ${ }^{2}$ \\ ${ }^{1}$ Department of Physics and Astronomy, University of Leicester, University Road, Leicester, LE1 7RH, UK \\ 2 Max-Planck-Institut für Aeronomie, D-37189 Katlenburg-Lindau, Germany
}

Received: 25 September 1996 / Revised: 6 January 1997 / Accepted: 7 January 1997

\begin{abstract}
The CUTLASS Finland HF radar has been operated in conjunction with the EISCAT Tromsø RF ionospheric heater facility to examine a ULF wave characteristic of the development of a field line resonance (FLR) driven by a cavity mode caused by a magnetospheric impulse. When the heater is on, striating the ionosphere with field-aligned ionospheric electron density irregularities, a large enough radar target is generated to allow post-integration over only 1 second. When combined with $15 \mathrm{~km}$ range gates, this gives radar measurements of a naturally occurring ULF wave at a far better temporal and spatial resolution than has been achieved previously. The time-dependent signature of the ULF wave has been examined as it evolves from a large-scale cavity resonance, through a transient where the wave period was latitude-dependent and the oscillation had the characteristics of freely ringing field lines, and finally to a very narrow, small-scale local field line resonance. The resonance width of the FLR is only $60 \mathrm{~km}$ and this is compared with previous observations and theory. The FLR wave signature is strongly attenuated in the ground magnetometer data. The characterisation of the impulse driven FLR was only achieved very crudely with the ground magnetometer data and, in fact, an accurate determination of the properties of the cavity and field line resonant systems challenges the currently available limitations of ionospheric radar techniques. The combination of the latest ionospheric radars and facilities such as the Tromsø ionospheric heater can result in a powerful new tool for geophysical research.
\end{abstract}

\section{Introduction}

The transient magnetohydrodynamic response to the impulsive excitation of the magnetospheric cavity has been the subject of a number of observational studies at

Correspondence to: T. K. Yeoman high latitudes (e.g., Nopper et al., 1982; Kivelson et al., 1984; Baumjohann et al., 1984; Allan et al., 1985; Potemra et al., 1989). Impulsive excitation of the magnetosphere has also recently been the subject of a considerable number of theoretical and modelling studies, principally concerned with the coupling of the field line resonance activity with the compressional mode cavity resonance activity (Allan et al., 1986a, b, 1987; Kivelson and Southwood, 1985, 1986; Inhester, 1987; Krauss-Varban and Patel, 1988; Zhu and Kivelson, 1988, 1989; Lee and Lysak, 1991; Lysak and Lee, 1992; Samson et al., 1992). In discussing these models the phrase "cavity mode" should in some cases be replaced by "wave guide mode", as an azimuthally travelling wave is assumed (Samson et al., 1992).

Up to the present some of the strongest experimental evidence for the existence of magnetospheric cavity/ wave guide modes has come from the inner magnetosphere, the plasmasphere. For example, Sutcliffe and Yumoto (1989; 1991), Yeoman and Orr (1989), Yumoto (1990), Yumoto et al. (1990), Lin et al. (1991), Yeoman et al. (1991) have all proposed that low- and midlatitude $\mathrm{Pi} 2$ pulsations are driven by compressional cavity resonances.

At high latitudes, Crowley et al. (1987) studied the damping of a ULF wave observed in ground magnetometer data and by the EISCAT radar, and concluded that energy was being fed into the field line resonance by a global cavity mode. Ruohoniemi et al. (1991) and Samson et al. (1992) have demonstrated that, on occasions, nightside and early morning data from the high latitude Goose Bay HF radar, Canada, showed structured spectra with distinct spectral peaks at a discrete set of frequencies. These were interpreted as field line resonances (FLRs) driven by magnetospheric cavity/wave guide modes. Similar structured spectra have recently been detected at sub-auroral latitudes by Provan and Yeoman (1996).

One highly characteristic aspect of the wave signatures predicted from a coupled cavity/wave guide - FLR system is the initial transient wave form. This transient 
occurs as coupling between the magnetosonic wave mode of the resonant cavity and the Alfvénic wave mode of the resonant field line leads to the irreversible transfer of energy from the cavity resonance to the field line resonance. McDiarmid and Allan (1990) produced a numerical simulation of the signature expected from such a coupled magnetospheric cavity/wave guide mode in auroral radar data, such as the Scandinavian Twin Auroral Radar Experiment (STARE, Greenwald et al., 1978) or the Sweden And Britain Radar-auroral Experiment (SABRE, Nielsen et al., 1983). McDiarmid and Allan (1990) noted that a latitude-dependent period occurred early in the wave packet, as the oscillation evolved from a cavity resonance, where phase is constant with latitude, to a FLR which has a characteristic phase change of $180^{\circ}$ in latitude. McDiarmid and Allan (1990) also noted that such wave characteristics were very difficult to derive from the simulated radar data.

A transient wave signature, reminiscent of the ULF waves simulated by McDiarmid and Allan (1990), was noted in the SABRE Wick radar data by McDiarmid et al. (1994). This oscillation was demonstrated to start as a compressional mode wave in spacecraft magnetometer data, and then to evolve into field line resonances in both the morning and afternoon sectors, although the wave structure was quite different in the pre- and post-noon regions.

In the present study, a high-latitude observation of a wave signature characteristic of a coupled cavity/wave guide - FLR is presented. This wave has been detected at very high spatial and temporal resolution by the Cooperative UK Twin Located Auroral Sounding System (CUTLASS) radar, running a SuperDARN discretionary mode experiment (Greenwald et al., 1995). The very high spatial and temporal resolution has been enabled through the generation of field-aligned electron density irregularities with the European Incoherent Scatter (EISCAT) high power RF ionospheric heating facility at Tromsø, in northern Norway (see e.g. Stubbe, 1996). These irregularities then act as an artificially produced target for the CUTLASS coherent scatter radar system.

\section{Instrumentation}

The ionospheric convection velocities in this study are provided by the CUTLASS Finland radar. CUTLASS is a bistatic HF coherent radar, with stations in Finland and Iceland, and forms part of the international SuperDARN chain of HF radars (Greenwald et al., 1995). Each radar of the system is a frequency agile (8-20 MHz) radar, routinely measuring the line-of-sight (1-o-s) Doppler velocity and spectral width of, and the backscattered power from, ionospheric plasma irregularities. The radars each form 16 beams of azimuthal separation $3.24^{\circ}$. Each beam is gated into 75 range bins, each of length $45 \mathrm{~km}$ in standard operations, when the dwell time for each beam is $7 \mathrm{~s}$, giving a full 16 beam scan, covering $52^{\circ}$ in azimuth and over $3000 \mathrm{~km}$ in range (an area of over $4 \times 10^{6} \mathrm{~km}^{2}$ ), every $2 \mathrm{~min}$. Common- volume data from the two stations can be combined to provide convection velocities perpendicular to the magnetic field, although no common volume backscatter was detected during the interval under study here. During this interval the Finland radar was operating a non-standard scan mode. In this mode, rather than the usual anticlockwise sweep through beams $15,14,13, \ldots, 0$ the Finland radar was confined to a single look direction, beam five. This beam overlies both the EISCAT radar facility and the ionospheric heater at Tromsø. The radar operations at this time also employed a range gating of $15 \mathrm{~km}$, the first gate being at a range of $480 \mathrm{~km}$, and an integration time of $1 \mathrm{~s}$. This arrangement was designed to explore the generation of field-aligned electron density irregularities at very high temporal and spatial resolution. This dataset is supplemented here by data from ground magnetometers, provided by the International Monitor for Auroral Geomagnetic Effects, IMAGE) (Lühr, 1994). These instruments are fluxgate magnetometers, with a sampling interval of $10 \mathrm{~s}$ for the interval under consideration here. The IMAGE stations employed in this study, and the location of the high resolution CUTLASS Finland Beam 5 are illustrated in Fig. 1.

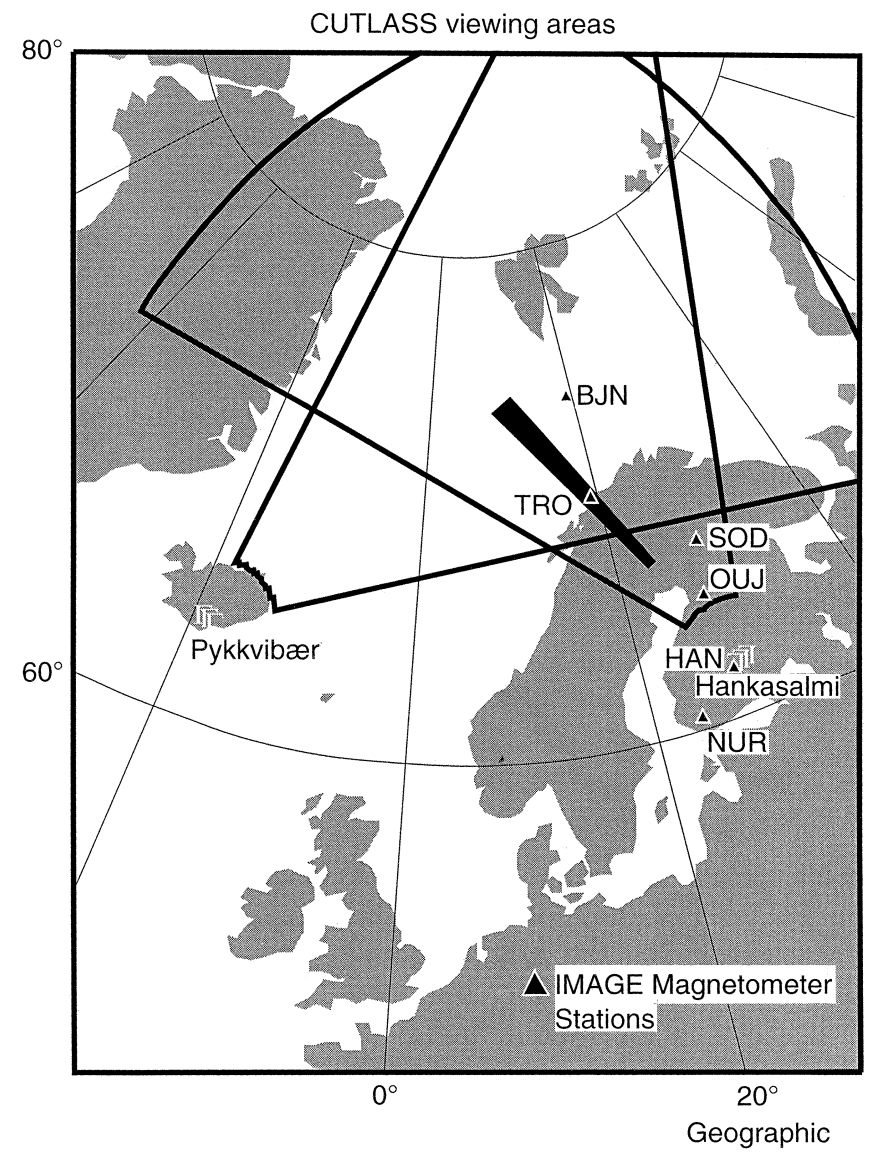

Fig. 1. Locations of the IMAGE magnetometer stations used in this study and the fields-of-view of the CUTLASS radars. The location of the CUTLASS Finland $15 \mathrm{~km}$ resolution single beam experiment is marked as a solid black region 


\section{Observations}

During the interval from 18 to 26 April 1996, a series of experiments were conducted with the EISCAT Incoherent Scatter radar, the Tromsø ionospheric heating facility, the CUTLASS HF radar and a number of low power HF diagnostics. One well-known result of artificial modification of the ionosphere with a high power RF facility such as the Tromsø heater is the generation of field-aligned ionospheric electron density irregularities (see Stubbe, 1996). One of the principal scientific aims of this experimental campaign was the delineation of the spatial and temporal development of these irregularities with the CUTLASS coherent scatter radar, which will backscatter from irregularities of wavelength of order 10-20 m, depending on the operating frequency. The results of these experiments will be presented elsewhere (T. R. Robinson, G. E. Bond, personal communication, 1996). Coherent backscatter from these artificially induced targets is recorded over a region where the heater power is sufficient to generate irregularities. The power backscattered is proportional to the mean square deviation of the electron density associated with the artificial irregularities. Backscatter signal levels obtained from artificial irregularities appear to be significantly higher than those from natural irregularities and this allows a reduction in the integration period of the radar. Integration periods of only one second have been possible, giving observations of regions of backscatter of $\sim 150 \mathrm{~km}$ extent in range with signal strengths in excess of $30 \mathrm{~dB}$.

During the experimental campaign, between 1600 and 1630 UT on 23 April, quite by chance, an opportunity arose to study a naturally occurring magnetospheric ULF wave within the region of artificially generated radar backscatter. The Tromsø heater was operating at frequencies between 3.90 and $4.01 \mathrm{MHz}$, and its interaction height in the ionosphere is estimated at $\sim 200 \mathrm{~km}$. Unfortunately, the EISCAT radar had ceased operation at $1600 \mathrm{UT}$. Although modification of the $\mathrm{D}$ and $\mathrm{E}$ region ionosphere at ULF frequencies can be used to artificially generate a magnetic response of up to $10 \mathrm{nT}$ (see Stubbe, 1996 and references therein), in the current experiment of F region heating the ULF wave is clearly of natural origin. Some evidence for observations of naturally occurring travelling ionospheric disturbances with HF diagnostics scattered by the Sura ionospheric heater has been presented by Blagoveshchenskaya and Troshichev (1996). They also observed wave activity in the ULF band, but attributed these to artificially generated waves. Although the experimental arrangement in the present study was optimised for plasma physics studies, the data collected offer a unique opportunity to study an important naturally occurring geophysical phenomenon with a very high spatial and temporal resolution. Figure 2 presents an overview of the radar and magnetometer data during the interval. A greyscale representation of the Finland line-of-sight velocity data is given in the top panel, with flow toward the radar (equatorward flow, positive Doppler velocity) plotted in light shading and flow away from the radar (poleward flow, negative Doppler velocity) plotted in dark shading. Patches of radar backscatter are observed, covering a spatial region of $\sim 1.5^{\circ}$ of geomagnetic latitude (throughout this study altitude adjusted corrected geomagnetic (AACGM) coordinates, based on Baker and Wing, 1989 are employed). The backscatter patches occur during periods when the Tromsø heater is operational (marked with vertical dashed lines). During this interval the heater operated the following sequence. A 2-min "tuning" period, during which the heater power was gradually increased, was followed by a 2-min "off" period. This was then followed by a 3-min full power heater "on" period, and then a 3 min "off". This sequence was repeated three times. Note that the artificial backscatter does not start immediately after the onset of a tuning cycle, as irregularities are only generated when the heater power has reached a threshold value. In addition, the artificial backscatter persists after the heater has been switched off, as the irregularities generated have a characteristic decay time. The latest results suggest that the heater-induced irregularities detected by CUTLASS decay to a level of $3 \mathrm{~dB}$ in $\sim 100 \mathrm{~s}$ (G. E. Bond, personal communication). The second panel presents a time series for a single range gate, gate 31 . Sections of the ULF wave forms can be clearly distinguished in this plot, with the background ionospheric drift velocity of $\sim 150 \mathrm{~m} \mathrm{~s}^{-1}$ being modulated by the ULF wave to values between $100 \mathrm{~m} \mathrm{~s}^{-1}$ and $300 \mathrm{~m} \mathrm{~s}^{-1}$. The ULF wave signature persists with a peak amplitude of between 100 and $200 \mathrm{~m} \mathrm{~s}^{-1}$ for the interval 1600-1624 UT. The lower four panels of Fig. 1 present the $X$ and $Y$ component magnetic field measurements from the Tromsø (TRO) magnetometer which lies underneath beam 5, gate 31 cell of the CUTLASS Finland radar in the discretionary radar programme running at this time. AULF wave can be seen both in the filtered and unfiltered data from TRO at a similar frequency to that observed in the radar data. The wave signature in the magnetometer data is strongest in the interval 1603-1613 UT. Fourier analysis of the wave signature at TRO reveals a dominant wave in the $X$ component with a period of $143 \mathrm{~s}(7 \mathrm{mHz})$.

The ULF wave in ground-based magnetic field measurements is examined in more detail in Fig. 3, which displays $X$ and $Y$ component magnetic field data from six IMAGE magnetometer stations, covering magnetic latitudes from $56.8^{\circ}-71.4^{\circ}$ geomagnetic latitude, bandpass filtered between 150 and $50 \mathrm{~s}$ (6.67$20.0 \mathrm{mHz}$ ). A coherent wave packet can be seen across the magnetometer chain, with maximum amplitude of $\sim 10 \mathrm{nT}$ (peak-to-peak) observed in the $X$ component magnetic field between the latitudes of TRO and SOD. Fourier power spectral analysis of the magnetometer data is presented in Fig. 4. This confirms that the dominant frequency is generally $7-8 \mathrm{mHz}$, and that the largest power at this frequency is found in the $X$ component at SOD and TRO. Examining the relative phase of the wave packets in Fig. 3 near the time of their maximum in amplitude, at 16:07 UT, the $Y$ components can be seen to be approximately in phase across the stations. In contrast, a phase change in excess of $180^{\circ}$ 
SUPERDARN / IMAGE 23 April 1996

CUTLASS Finland and Troms $\varnothing$

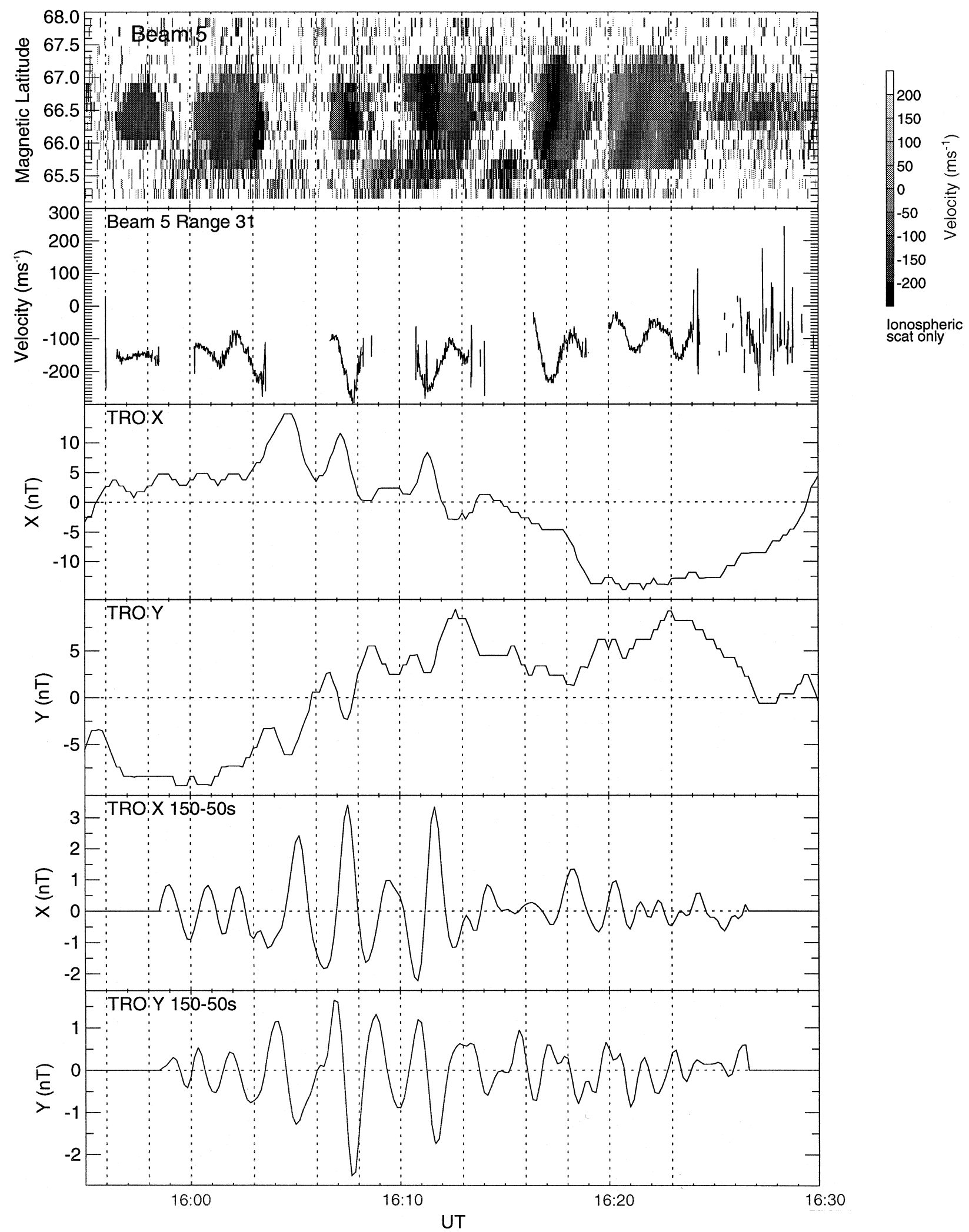

Fig. 2. An overview of the radar and magnetometer data during the interval. A greyscale representation of the Finland line-of-sight velocity data is given in the top panel (positive velocities are towards the radar). Patches of radar backscatter are observed during periods when the Troms $\varnothing$ ionospheric heater is operational (marked with vertical dashed lines). The second panel plots a time series for a single range gate, gate 31, which overlies the Tromsø magnetometer. Filtered $(150-50 \mathrm{~s}$, $6.67-20 \mathrm{mHz}$ ) and unfiltered $X$ and $Y$ component magnetic field measurements are presented in the lower four panels. A correlated ULF wave signature can be seen in data from both instruments 
IMAGE X component 23 April 1996

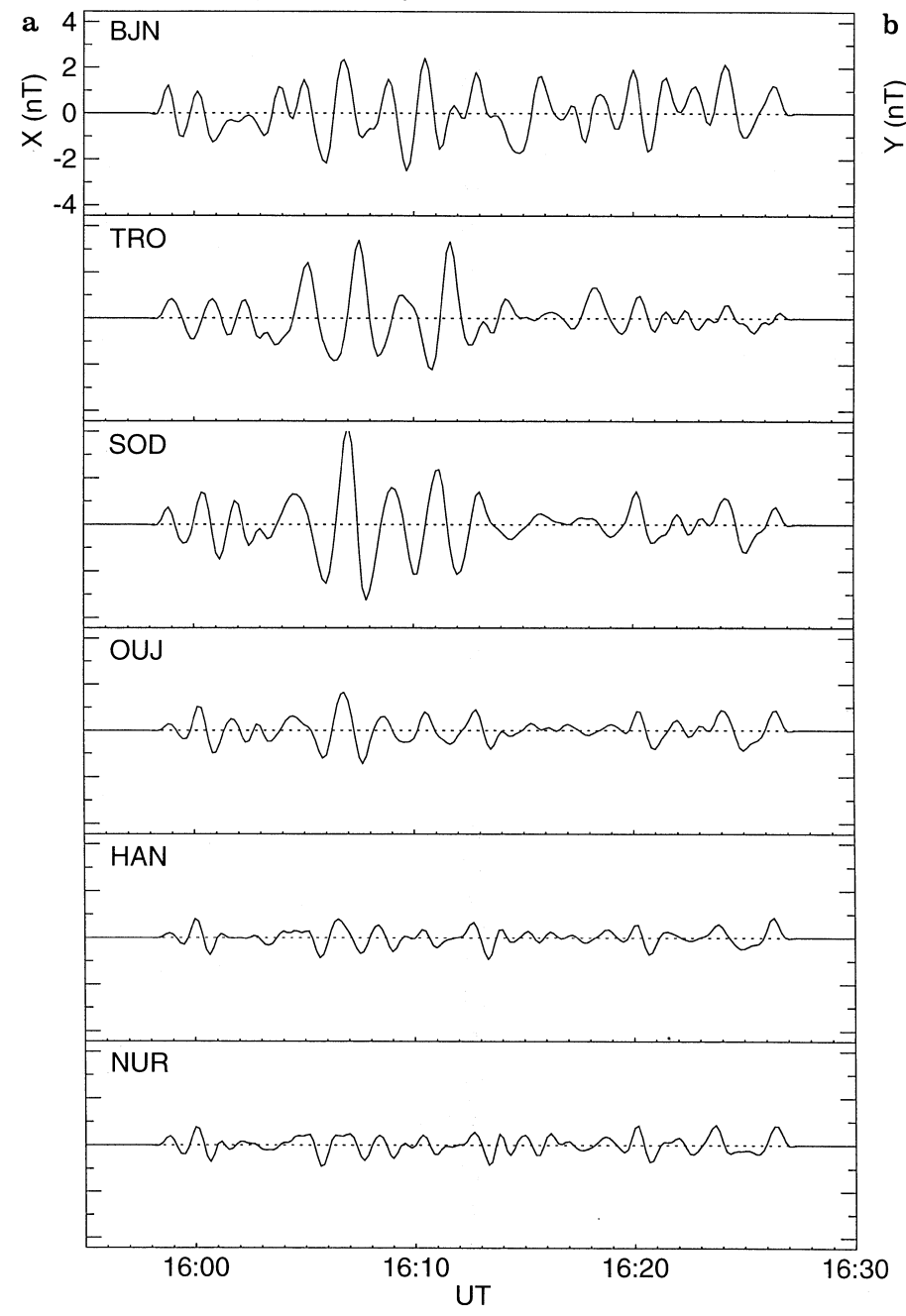

IMAGE Y component 23 April 1996

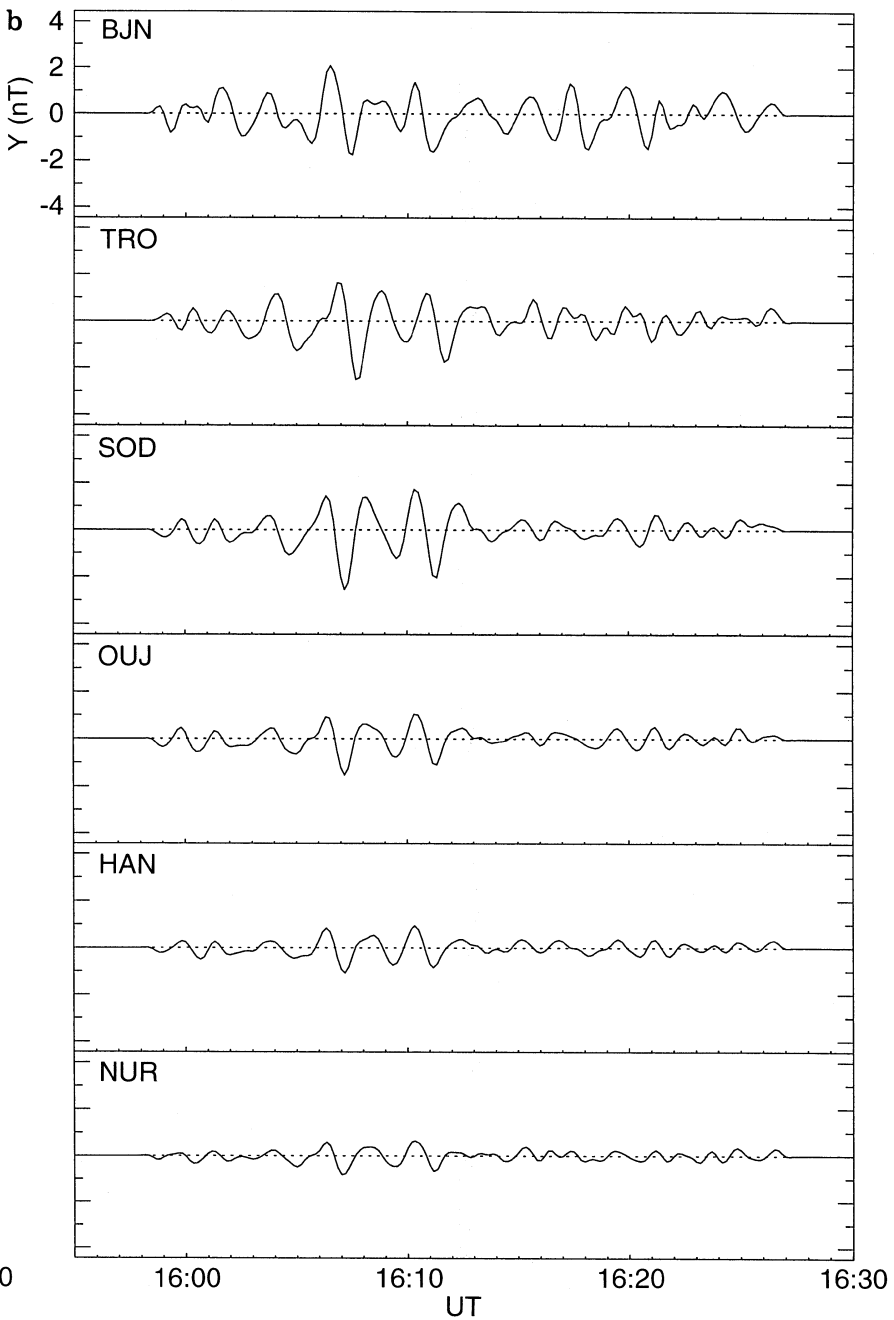

Fig. 3 a, b. Magnetic field data from six IMAGE magnetometer stations, bandpass filtered between 150 and $50 \mathrm{~s}(6.67-20.0 \mathrm{mHz})$, for a the $X$ component and $\mathbf{b}$ the $Y$ component

can be seen in the $X$ component field measurements, with the lower latitude stations leading in phase.

The latitude variation of the line-of-sight CUTLASS data is displayed in Fig. 5. Stacked time series of Finland line-of-sight velocity data for nine $15 \mathrm{~km}$ range gates (gates 28-36, ranges 900-1035 km) are included. Although the analysis of the ULF wave, which has a period of $\sim 2 \mathrm{~min}$, is limited by the heater cycle, which restricts the available data to 2 - and 3 - min sections, the very high temporal and spatial resolution of the data enable a detailed picture of the wave parameters to be obtained for a number of intervals during the wave packet. The ULF wave signature is well defined over the $135 \mathrm{~km}$ of latitude illuminated by the Tromsø heater. The wave is of a fairly constant amplitude over the nine range gates early in the wave packet, but has a peak amplitude in the equatormost range gates by 1620 UT. The early wave cycles are also in phase over the nine range gates, whereas by the later parts of the wave packet a phase change of $\sim 180^{\circ}$ can be seen over the $135 \mathrm{~km}$ separation, with the lower latitude range gates leading in phase. The characteristics of these wave packets in the radar data are presented in detail in Fig. 7.

The spatial and temporal variation of the wave parameters in the ground magnetometer and ionospheric drift velocity measurements will now be examined in more detail. In Fig. 6 wave parameters derived from the magnetometer data presented in Fig. 3 are examined. Panels $\mathrm{a}$ and $\mathrm{b}$ present the amplitude and phase of the dominant Fourier component of the $X$ and $Y$ component magnetometer data. The magnetic field data for the entire ground-observed wave packet, between 1557 and $1618 \mathrm{UT}$, bandpass filtered between 150 and $100 \mathrm{~s}$ $(6.67-10.0 \mathrm{mHz})$ has been included in the Fourier analysis, and the average frequency of peak spectral power over the array is $7.9 \mathrm{mHz}$. The spectral amplitude and phase at this frequency are presented. Analysis of this spectral component reveals a pattern typical of a classical FLR signature. There is a clear peak in spectral power in latitude, in this case at SOD, with the $X$ component the dominant component in the ground magnetic field. The spectral phase is fairly constant in latitude for the $Y$ component magnetic field, but has a 

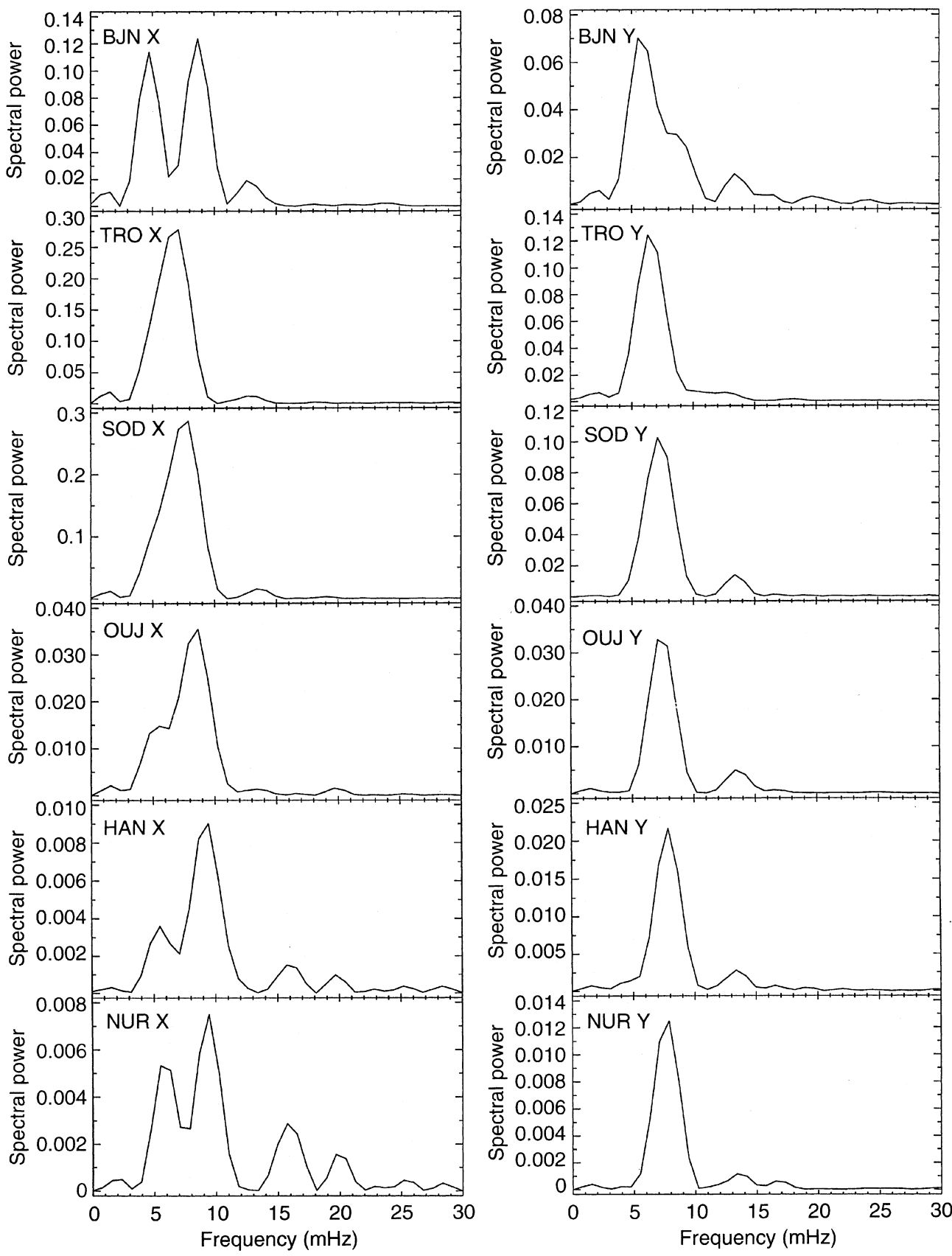

Fig. 4. Fourier power spectral analysis (linear arbitrary units) for the magnetometer data presented in Fig. 3, in this case detrended with a $300 \mathrm{~s}(3.3 \mathrm{mHz})$ high pass filter clear lead at the lower latitude stations in the $X$ component. The latitudinal phase change in the $X$ component measured here is, however, almost $360^{\circ}$, rather than the $180^{\circ}$ predicted by field line resonance theory (Southwood, 1974; Chen and Hasegawa, 1974). The horizontal shaded bar shows the latitudinal extent of the radar data presented in Figs. 5 and 7.

Figure 7 presents wave parameters derived from the CUTLASS radar line-of-sight velocity data. Following McDiarmid and Allan (1990), wave parameters are calculated directly in the time domain from the velocity measurements, with the time shifts between the wave peaks and troughs being used to derive wave period and phase. This technique was found to be the most useful by McDiarmid and Allan (1990) and, in addition, allows the effect of the heater cycle to be deconvolved from the wave parameter analysis. In each panel, wave parameters have been derived for five short sections of the CUTLASS velocity timeseries during heater "on" intervals at 1601-1603, 1606-1608, 1611-1613, 1616-1618, and 1621-1622 UT. Figure 7a presents the calculated wave periods. There is, in general, no clear variation of wave period with latitude, but the profile measured at 1611-1613 UT shows the strongest latitude variation. The wave phase, relative to the lowest latitude range gate available for each time interval is shown in Fig. 7b. This analysis clearly shows the evolution of the wave packet from a latitudinal sequence of in-phase time series from 1601-1613 UT to a sequence displaying the characteristic latitudinal phase variation of a FLR (Southwood, 


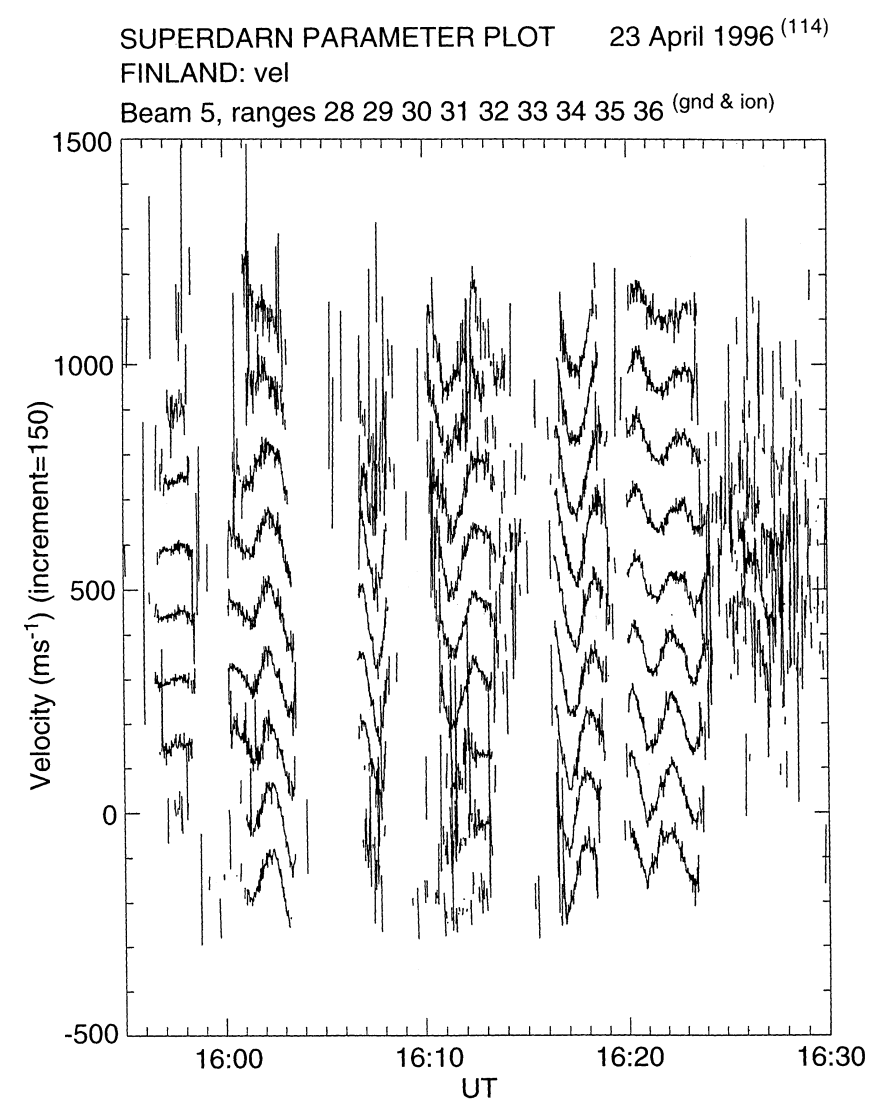

Fig. 5. Stacked time series of Finland line-of-sight velocity data for nine $15 \mathrm{~km}$ range gates (gates 28-36, ranges 900-1035 km). Range gates $28-35$ are used to derive the wave parameters in Fig. 7

1974; Chen and Hasegawa, 1974). Figure 7c displays the peak-to-peak wave amplitude. Again a clear evolution can be seen during the wave packet, from a latitude- independent profile early in the oscillation to a sharply peaked resonant structure at $66.25^{\circ}$ geomagnetic latitude. This transition appears to be surprisingly sharp, occurring between 16:13 and 16:16 UT, although this may, in part, be an artefact of the observational constraints imposed by the heater cycle.

Wave period estimates have also been derived from the ground magnetometer data using the technique employed for Fig. 7. Figure 6c presents the dominant wave period in the horizontal ground magnetic field data for two intervals during the wave event, one early in the wave packet (1605-1608 UT) and one late in the ground-observed wave packet (1608-1612 UT). The wave period can be seen to have a distinct latitudinal variation, with the field oscillating at a longer period at higher latitudes in the early part of the ground magnetometer recordings. However, this trend becomes less apparent as the wave packet develops.

\section{Discussion}

The combination of the Tromsø heater and the CUTLASS Finland HF coherent scatter radar has enabled a high temporal and spatial resolution study of a highlatitude ULF wave. The high resolution study possible with the radar data has been compared with an analysis of the lower resolution, but more spatially extensive, magnetometer data. The wave has the characteristics of the transient signature of a coupled cavity/waveguide FLR driven by an impulse on the magnetopause. The period of the observed wave suggests that it is a harmonic of the local field line eigenperiod (Poulter et al., 1984). A similar wave observed by McDiarmid et al. (1994) was demonstrated to be most probably a second harmonic. The observed wave signatures can

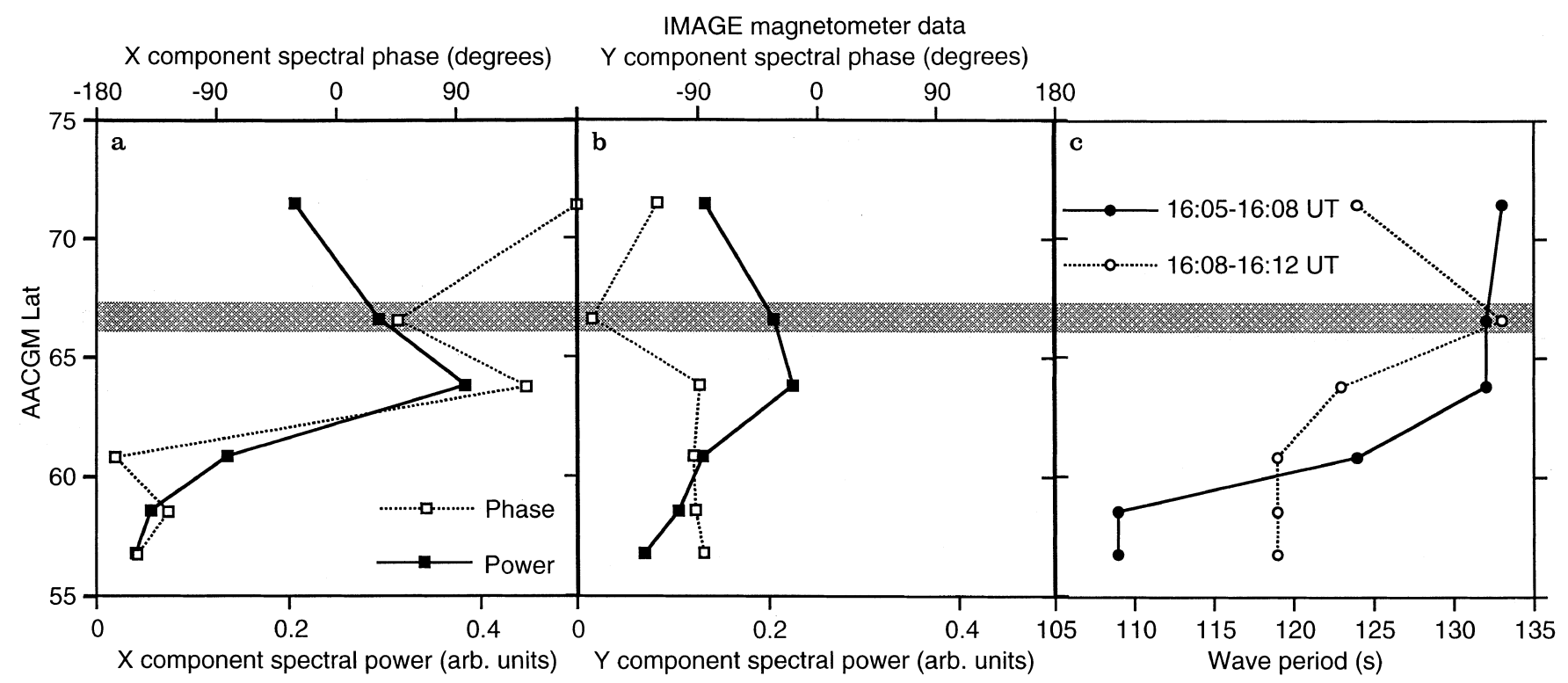

Fig. 6 a-c. Wave parameters derived from the magnetometer data presented in Fig. 3. Fourier spectral amplitude and phase at $7.9 \mathrm{mHz}$ are presented for a the $X$ component and b the $Y$ component. c Shows the dominant wave period for two intervals during the wave event (see text for details). The horizontal shaded bar shows the latitudinal extent of the radar data presented in Fig. 5 and 7 


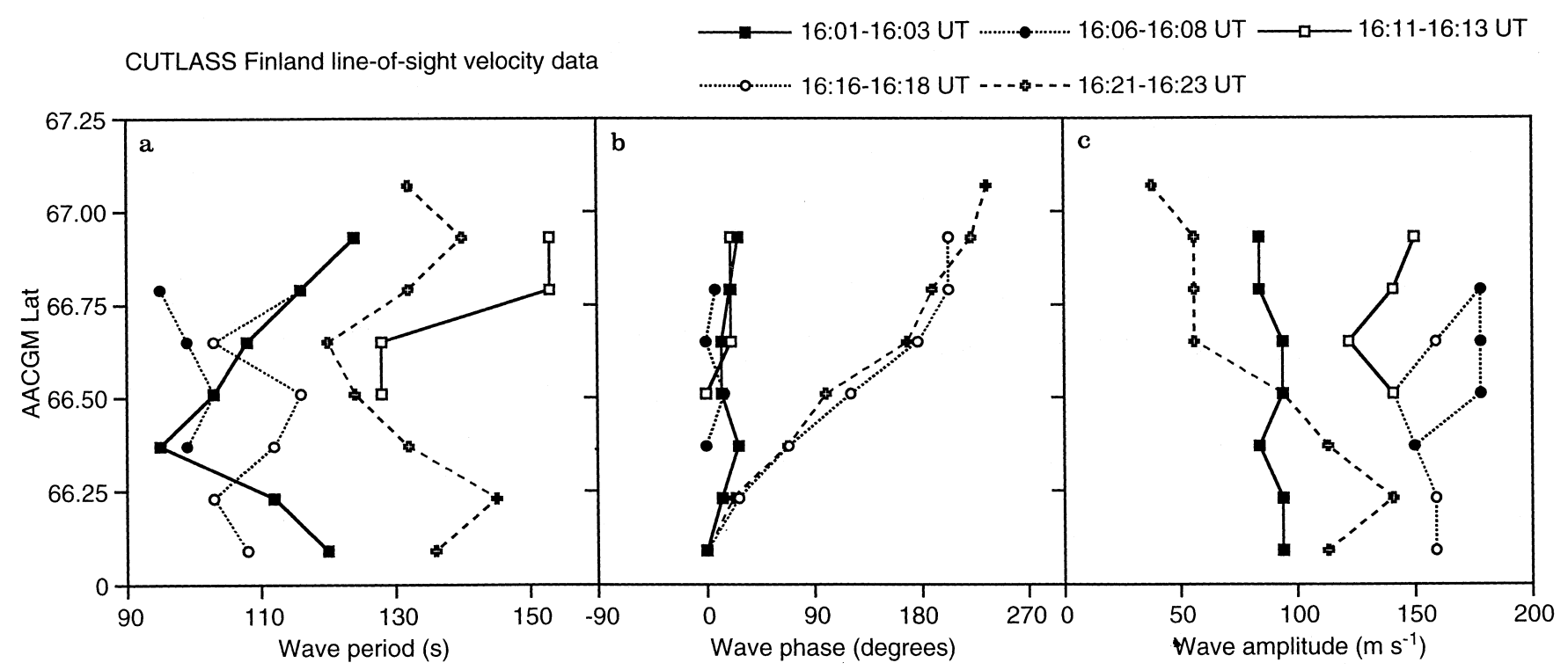

Fig. 7 a-c. Wave parameters derived from the CUTLASS radar lineof-sight velocity data presented in Fig. 5. a Wave periods, b wave phase relative to the lowest latitude range gate and $\mathbf{c}$ peak-to-peak

most readily be compared with the simulation of an impulse driven field line resonance in VHF coherent radar data presented by McDiarmid and Allan (1990). They used the output of the time-dependent numerical model of Allan et al. (1986b) to model the development of a FLR driven by a cavity mode caused by a magnetospheric impulse. The most characteristic signature of these simulations was the evolution of the response from the constant period-constant phase characteristic of a cavity resonance, through a transient, latitude-dependent frequency region, where each field line oscillated at its natural frequency, to a resonant signature with an amplitude peak accompanied by a $180^{\circ}$ phase change in latitude, as the energy of the cavity/waveguide mode was channelled into a FLR. The simulation showed that the latitude-dependent pulsation period was observed most strongly in the early wave cycles of the oscillation, and had almost entirely disappeared by the latter half of the modelled wave. Another important result of this modelling was the prediction that even with the good spatial $(20 \mathrm{~km})$ and temporal (20 s) resolution of the STARE/SABRE type radars, the transient features of these resonances would be difficult to observe. The difficulty in making accurate observational studies of such wave phenomena is clearly illustrated in the contrast between Fig. 6 and 7. The magnetometer data suggest a latitudinally broad, resonant structure. However, this is a consequence of the temporal evolution of the signal during the wave packet and the spatial integration of the ground magnetometers (see Sect. 4.3). The radar data reveals that the resonance is actually confined to a very narrow region (Fig. 7; the latitude region of this figure is indicated in Fig. 6 by the horizontal shaded bar). The oscillation will be discussed in detail in three sections: (a) the initial cavity oscillation, (b) the coupled transient and (c) the driven field line resonance. wave amplitude. Each panel presents parameters derived from five sub-intervals of the wave packet at 1601-1603, 1606-1608, 1611-1613, 1616-1618, and 1621-1623 UT (see text for details)

\subsection{The cavity resonance}

The initial wave cycles of the transient pulsation observed here are most characteristic of the impulsedriven cavity/wave guide resonance. The amplitude and phase are relatively constant with latitude, as expected for a large-scale cavity resonance. This section of the wave packet appears strongly in the ground magnetometer data. The early wave packet does not have a simple damped envelope, however, as might be expected for an impulse-driven resonance. Rather it grows between 1601 and 1608 UT in both the ground magnetometer data and the ionospheric velocity data, before damping and coupling to a FLR reduce the amplitude.

\subsection{The coupled transient}

The transition between a cavity resonance-like and a FLR-like signature is characterised by a latitude-dependent period. Latitude-dependent periods, due to magnetospheric field lines freely ringing at their natural frequencies have been observed at high latitude by Poulter and Nielsen (1982) and Allan et al. (1985). In the present study this transition region can be seen in the early part of the ground-observed oscillation from 16051608 (Fig. 6c), where the wave period varies from 110$130 \mathrm{~s}$. The later parts of the wave form give unclear results. The results from the analysis of the ionospheric velocity time series do not show a clear dependence of period with latitude, although a latitude variation of $130-150 \mathrm{~s}$ is observed from 1611-1613 UT.

\subsection{The field line resonance}

The resonant field line section of the oscillation modelled by McDiarmid and Allan (1990) has a 
resonance width of $\sim 0.6^{\circ}$ latitude $(67 \mathrm{~km})$ and a phase slope of $160-195^{\circ} /{ }^{\circ}$ latitude. In the simulated radar data most appropriate for HF radars, with no velocity threshold effects, this appeared as a phase slope of $110^{\circ}{ }^{\circ}$ lat., with a $0.8^{\circ}$ latitude $(90 \mathrm{~km})$ width. Probably the best observational candidates for such a wave previously reported were the oscillations studied by McDiarmid and Nielsen (1987) and McDiarmid et al. (1994). In the present study, using a radar experiment of spatial resolution $15 \mathrm{~km}$, slightly superior but comparable to the VHF radars, but with much improved temporal resolution (1s rather than 20s), a peak phase slope of $270^{\circ}{ }^{\circ}$ Lat. is observed and the full width at half maximum (FWHM) of the resonance can be estimated at $0.54^{\circ}$ Latitude or $60 \mathrm{~km}$. Previous observations of the widths of field line resonances using the STARE radar (20 km, $20 \mathrm{~s}$ resolution, Walker et al., 1979) and the Goose Bay HF radar $(45$ km, 96 s resolution, Walker et al., 1992) gave results of FWHM $\sim 100 \mathrm{~km}$. McDiarmid et al. (1994) inferred a resonance width of $2^{\circ}$ latitude $(220 \mathrm{~km})$ for their observation of an impulsedriven FLR.

The theoretical minimum FLR width in the magnetospheric equatorial plane was evaluated as $0.005 L R_{E}$ by Newton et al. (1978), corresponding to $\sim 6 \mathrm{~km}$ in the ionosphere at the latitude of Tromsø, where a separation of $100 \mathrm{~km}$ in the ionosphere will map to $0.59 R_{E}$ in the magnetospheric equatorial plane. This calculation assumed a light damping due to ionospheric Joule dissipation only, with the height integrated Pedersen conductivity, $\sum_{P}=10 \mathrm{~S}$. Any additional damping effects will act to widen the resonance.

In the present study, although the EISCAT radar ceased to operate before the occurrence of the ULF wave, at 16:00 UT, data from 15:00-16:00 UT show that the height-integrated Hall and Pedersen conductivites, $\sum_{H}$ and $\sum_{P}$, both had a steady value of $3 \mathrm{~S}$. It thus seems reasonable to take this value as appropriate for the wave from 16:00-16:30 UT. These values have been calculated from the EISCAT alternating code electron density profiles in the field-aligned pointing position, along with the IGRF magnetic field model and the MSIS86 model of the neutral atmosphere. The conductivities have been height-integrated between 90 and $200 \mathrm{~km}$. A value of $\sum_{P}=3 \mathrm{~S}$ implies a resonance width in the ionosphere of $30 \mathrm{~km}$ and $9 \mathrm{~km}$ respectively for a fundamental and second harmonic toroidal mode (based on the curves of Newton et al., 1978). These narrow theoretical resonance widths suggest that damping mechanisms other than ionospheric Joule dissipation are important in the wave event under study. A similar conclusion was reached for an impulse-driven wave by McDiarmid et al. (1994). Recently, Mann et al. (1995) have suggested that the asymptotic width of a field line resonance coupled to a magnetospheric cavity mode is in fact determined by the damping of the cavity mode rather than the resonant field line, and predicted a resonance width of $0.4 R_{E}$ in the magnetospheric equatorial plane at $L=7$ (corresponding to $68 \mathrm{~km}$ in the ionosphere above Tromsø). This value very closely matches the observations presented here.
It is difficult to confirm these calculations with a direct estimate of the wave damping from the observed time series. For a coupled cavity mode a damping rate of lower than that due to ionospheric Joule dissipation will be inferred, if the cavity mode is still driving the field line resonance. In addition the difficulty in estimating damping rates of transient pulsations from ground magnetic field data has been demonstrated in the modelling of Poulter and Allan (1985). In the example studied here, clearly the ground magnetometer data would give a very deceptive estimate of damping rate. Unfortunately in this case, the driven field line resonance is not detected for long enough for the ionospheric radar data to provide a good estimate of the damping either.

The field line resonance part of the oscillation, although clearly delineated in the radar data, is not apparent in the ground magnetometer data. For such a narrow resonance this is perhaps not surprising. Ground magnetometer signals are attenuated at small-scale lengths, due to the spatial integration of the $\mathrm{E}$ region current systems responsible for the magnetic signature over an area of the ionosphere of radius of the same order of the E region height. Hughes and Southwood (1976) demonstrated that a signal attenuation of $\sim 1$ order of magnitude occurred if the azimuthal scale length of the ULF wave was $50 \mathrm{~km}$. Attenuation increases of a factor of 5 were observed by Yeoman et al. (1992) in a comparison of pulsations in ionospheric radar and ground magnetometer data as the azimuthal wave number, $m$, increased from 3 to 18 .

\section{Summary}

AULF wave characteristic of the development of a FLR driven by a cavity mode caused by a magnetospheric impulse has been investigated by means of a novel experimental technique. The time-dependent signature of the waveform has been examined as it evolves from a large-scale cavity resonance, through a transient where the wave period was latitude-dependent and the oscillation had the characteristics of freely ringing field lines, and finally to a very narrow, small-scale local field line resonance. The FLR was only $60 \mathrm{~km}$ in width and was highly attenuated in the ground magnetometer data.

The characterisation of impulse driven FLRs can only be achieved very crudely by ground magnetometer arrays and, in fact, an accurate determination of the properties of the cavity and field line resonant systems challenges the currently available limitations of ionospheric radar techniques.

The results reported here demonstrate that the combination of the latest ionospheric radars and an ionospheric heater, such as that at Tromsø, provide a powerful new tool for active geophysical research.

Acknowledgements. The IMAGE magnetometer data were kindly supplied by Lasse Häkkinen of the Finnish Meteorological Institute (FMI). The CUTLASS HF radars are deployed and operated by the University of Leicester, and are jointly funded by the UK Particle Physics and Astronomy Research Council, the 
Finnish Meteorological Institute, and the Swedish Institute for Space Physics. The ionospheric modification facility at Tromsø is operated by the EISCAT Scientific Association, and is supported by the Suomen Akatemia (Finland), the Centre National de la Recherche Scientifique (France), the Max-Planck-Gesellschaft (Germany), the Norges forskningsråd (Norway), the Naturveterskapliga Forskningsrådet (Sweden) and the Particle Physics and Astronomy Research Council (United Kingdom).

Topical Editor K.-H. Glaßmeier thanks R. A. Greenwald and J. C. Samson for their help in evaluating this paper.

\section{References}

Allan, W., E. M. Poulter, K.-H. Glaßmeier, and H. Junginger Spatial and temporal structure of a high-latitude transient ULF pulsation, Planet. Space Sci. 33, 59-173, 1985.

Allan, W., S. P. White, and E. M. Poulter, Impulse-excited hydromagnetic cavity and field-line resonance in the magnetosphere, Planet. Space Sci., 34, 371-385, 1986a.

Allan, W., E. M. Poulter, and S. P. White, Hydromagnetic wave coupling in the magnetosphere - plasmapause effects on impulse-excited resonance, Planet. Space Sci., 34, 1189-1200, 1986b.

Allan, W., E. M. Poulter, and S. P. White, Hydromagnetic wave coupling in the magnetosphere - magnetic fields and Poynting fluxes, Planet. Space Sci., 35, 1181-1192, 1987.

Baker, K. B., and S. Wing, A new magnetic coordinate system for conjugate studies at high latitudes, J. Geophys. Res., 94, 91399143, 1989.

Baumjohann, W., H. Junginger, G. Haerendel, and O. H. Bauer, Resonant Alfvén waves excited by a sudden impulse, J. Geophys. Res., 89, 2765-2769, 1984.

Blagoveshchenskaya, N. F., and O. A. Troshichev, Ionospheric phenomena produced by modification experiments, J. Atmos. Terr. Phys., 58, 397-406, 1996.

Chen, L., and A. Hasegawa, A theory of long period magnetic pulsation 1. steady-state excitation of a field line resonance, J. Geophys. Res., 79, 1024-1032, 1974.

Crowley, G., W. J. Hughes, and T. B. Jones, Observational evidence of cavity modes in the Earth's magnetosphere, J. Geophys. Res., 92, 12 233-12 240, 1987.

Greenwald, R. A., K. B. Baker, J. R. Dudeney, M. Pinnock, T. B. Jones, E. C. Thomas, J.-P. Villain, J.-C. Cerisier, C. Senior, C. Hanuise, R. D. Hunsucker, G. Sofko, J. Koehler, E. Nielsen, R. Pellinan, A. D. M. Walker, N. Sato, and H. Yamagishi, DARN/ SuperDARN: a global view of the dynamics of high-altitude convection, Space Sci. Rev., 71, 761-796, 1995.

Greenwald, R. A., W. Weiss, E. Nielsen, and N. R. Thomson, STARE, A new radar auroral backscatter experiment in northern Scandinavia, Radio Sci., 13, 1021-1036, 1978.

Hughes, W. J., and D. J. Southwood, An illustration of modification of geomagnetic pulsation structure by the ionosphere, J. Geophys. Res., 81, 3241-3247, 1976.

Inhester, B., Numerical modelling of hydromagnetic wave structure in the magnetosphere, J. Geophys. Res., 92, 4751-4756, 1987.

Kivelson, M. G. and D. J. Southwood, Resonant ULF waves: a new interpretation, Geophys, Res. Lett., 12, 49-52, 1985.

Kivelson, M. G., and D. J. Southwood, Coupling of global magnetosphere MHD eigenmodes to field line resonances, J. Geophys. Res., 91, 4345-4351, 1986.

Kivelson, M. G., J. Etcheto, and J. G. Trotignon, Global compressional oscillations of the terrestrial magnetosphere: the evidence and a model, J. Geophys. Res., 89, 9851-9856, 1984.

Krauss-Varban, D., and V. L. Patel, Numerical analysis of the coupled hydromagnetic wave equations in the magnetosphere, J. Geophys. Res., 93, 9721-9729, 1988.

Lee, D.-H., and R. L. Lysak, Impulsive excitation of ULF waves in the three-dimensional dipole model: the initial results, J. Geophys. Res., 96, 3479-3486, 1991.
Lin, C. A., L. C. Lee, and Y. L. Sun, Observation of Pi2 pulsations at very latitude $(\mathrm{L}=1.06)$ station and magnetospheric cavity resonance, J. Geophys. Res., 96, 21 105-21 113, 1991.

Lühr, H., The IMAGE magnetometer network, STEP Int. Newsl., 4 (10), 4-6, 1994.

Lysak, R. L., and D.-H. Lee, Response of the dipole magnetosphere to pressure pulses, Geophys. Res. Lett., 19, 937-940, 1992.

Mann, I. R., A. N. Wright, and P. S. Cally, Coupling of magnetospheric cavity modes to field line resonances: a study of resonance widths, J. Geophys. Res., 100, 19 441-19 456, 1995.

McDiarmid, D. R., and W. Allan, Simulation and analysis of auroral radar signatures generated by a magnetospheric cavity mode, J. Geophys. Res., 95, 20 911-20 922, 1990.

McDiarmid, D. R., E. Nielsen, Simultaneous observation of monochromatic and variable period geomagnetic pulsations, $J$. Geophys. Res., 92, 4449-4457, 1987.

McDiarmid, D. R., T. K. Yeoman, I. F. Grant, and W. Allan, simultaneous observation of a travelling vortex structure in the morning sector and a field line resonance in the postnoon sector, J. Geophys. Res., 99, 8891-8904, 1994.

Newton, R. S., D. J. Southwood, and W.J. Hughes, Damping of geomagnetic pulsations by the ionosphere, Planet. Space Sci., 26, 201-209, 1978.

Nielsen, E., W. Guttler, E. C. Thomas, C. P. Stewart, T. B. Jones, and A. Hedburg, SABRE - a new radar auroral backscatter experiment, Nature, 304, 712-714, 1983.

Nopper, R. W., W. J. Hughes, C. G. Maclennan, and R. L. McPherron, Impulse-excited pulsations during the July 29, 1977 event, J. Geophys. Res., 87, 5911-5916, 1982.

Potemra, T. A., H. Lühr, L. J. Zanetti, K. Takahashi, R. E. Erlandson, G. T. Marklund, L. P. Block, L. G. Blomberg, and R. P. Lepping, Multisatellite and ground-based observations of transient ULF waves, J. Geophys. Res., 94, 2543-2554, 1989.

Poulter, E. M., and W. Allan, Transient ULF pulsation decay rates observed by ground based magnetometers: the contribution of spatial integration Planet. Space Sci., 33, 607-616, 1985.

Poulter, E. M., W. Allan, J. G. Keys, and E. Nielsen, Plasmatrough ion mass densities determined from ULF pulsation eigenperiods, Planet. Space Sci., 32, 1069-1078, 1984.

Poulter, E. M., and E. Nielsen, The hydromagnetic oscillation of individual shells of the geomagnetic field, J. Geophys. Res., 87, $10432-10438,1982$.

Provan, G., and T. K. Yeoman, A comparison of field line resonances observed at the Goose Bay and Wick radars, Ann. Geophysicae, 15, 231-235, 1977.

Ruohoniemi, J. M., R. A. Greenwald, K. B. Baker, and J. C. Samson, HF radar observations of Pc5 field line resonances in the midnight/early morning MLT sector, J. Geophys. Res., 96, 15 697-15 710, 1991.

Samson, J. C., B. G. Harrold, J. M, Ruohoniemi, R. A. Greenwald, and A. D. M. Walker, Field line resonances associated with MHD wave guides in the magnetosphere, Geophys. Res. Lett., 19, 441-444, 1992.

Southwood, D. J., Some features of field line resonances in the magnetosphere, Planet Space Sci., 22, 483-491, 1974

Stubbe, P., Review of ionospheric modification experiments at Tromsø, J. Atmos. Terr. Phys., 58, 349-368, 1996

Sutcliffe, P. R., and K. Yumoto, Dayside Pi2 pulsations at low latitude, Geophys. Res., Lett., 16, 887-890, 1989.

Sutcliffe, P. R., and K. Yumoto, On the cavity mode nature of lowlatitude Pi2 pulsations, J. Geophys. Res., 96, 1543-1551, 1991.

Walker, A. D. M., R. A. Greenwald, W. F. Stuart, and C. A. Green, STARE radar observations of Pc5 geomagnetic pulsations, J. Geophys. Res., 84, 3373-3388, 1979.

Walker, A. D. M., J. M. Ruohoniemi, K. B. Baker, R. A. Greenwald, and J. C. Samson, Spatial and temporal behavior of ULF pulsations observed by the Goose Bay HF radar, J. Geophys. Res., 97, 12 187-12 202, 1992. 
Yeoman, T. K., and D. Orr. Phase and spectral power of midlatitude Pi2 pulsations: evidence for a plasmaspheric cavity resonance, Planet. Space Sci., 37, 1367-1383, 1989.

Yeoman, T. K., M. Lester, D. K. Milling, and D. Orr, Polarization, propagation and MHD wave modes of Pi2 pulsations: SABRE/ SAMNET results, Planer. Space Sci., 39, 983-998, 1991.

Yeoman, T. K., Mao Tian, M. Lester, and T. B. Jones, A study of Pc5 hydromagnetic waves with equatorward phase propagation, Planet. Space Sci., 40, 797-810, 1992.

Yumoto, K., Evidence of magnetospheric cavity Pi2 waves, J. Geomag. Geoelectr., 42, 1281-1290, 1990.
Yumoto, K., K. Takahashi, T. Sakurai, P. R. Sutcliffe, S. Kokubun, H. Lühr, T. Saito, M. Kuwashima, and N. Sato, Multiple ground-based and satellite observations of global Pi2 magnetic pulsations, J. Geophys. Res., 95, 15 175-15 184, 1990.

Zhu, X. M., and M. G. Kivelson, Analytic formulation and quantitative solutions of the coupled ULF wave problem, J. Geophys. Res., 93, 8602-8612, 1988.

Zhu, X., and M. G. Kivelson, Global mode ULF pulsations in a magnetosphere with a non-monotonic Alfvén velocity profile, J. Geophys. Res., 94, 1479-1485, 1989. 